\title{
Spin filters with Fano dots
}

\author{
M. E. Torio, ${ }^{1}$ K. Hallberg, ${ }^{2}$ S. Flach,${ }^{3}$ A. E. Miroshnichenko, ${ }^{3}$ and M. Titov ${ }^{3,4}$ \\ ${ }^{1}$ Instituto de Física Rosario, CONICET-UNR, Bv. 27 de Febrero 210 bis, 2000 Rosario \\ ${ }^{2}$ Centro Atómico Bariloche and Instituto Balseiro, \\ Comisión Nacional de Energía Atómica, 8400 Bariloche, Argentina \\ ${ }^{3}$ Max-Planck-Institut für Physik komplexer Systeme, \\ Nöthnitzer Strasse 38, D-01187 Dresden, Germany \\ ${ }_{4}^{4}$ Physics Department, Columbia University, New York, NY 10027, USA
}

(Dated: June 7, 2018)

\begin{abstract}
We compute the zero bias conductance of electrons through a single ballistic channel weakly coupled to a side quantum dot with Coulomb interaction. In contrast to the standard setup which is designed to measure the transport through the dot, the channel conductance reveals Coulomb blockade dips rather then peaks due to the Fano-like backscattering. At zero temperature the Kondo effect leads to the formation of broad valleys of small conductance corresponding to an odd number of electrons on the dot. By applying a magnetic field in the dot region we find two dips corresponding to a total suppression in the conductance of spins up and down separated by an energy of the order of the Coulomb interaction. This provides a possibility of a perfect spin filter.
\end{abstract}

PACS numbers: 72.15.Qm, 73.23.Ad, 72.25.-b

Keywords:

\section{INTRODUCTION}

In recent decades the electric transport through quantum dots (QD) has been extensively studied both theoretically and experimentally 1, 2, 3]. As the result a comprehensive picture of a big variety of underlying physical phenomena has emerged (See e.g. [4, 5] and references therein). Confinement of electrons in small quantum dots leads to the necessity of taking into account their Coulomb repulsion. At finite temperatures the main effect is the Coulomb blockade [6, 7], which manifests itself in the oscillations of the dot's conductance versus the gate voltage applied to the dot. At very low temperature in the Coulomb blockade regime the dot's conductance increases in so-called "odd" valleys, where the number of electrons on the dot remains odd. This is a signature of the mesoscopic Kondo effect 8, 9, 10], which has been experimentally observed 11, 12, 13. In recent years, several authors have studied the possibility of spin polarization in transport through QD's 14, 15, 16, 17] and other nanoscopic configurations like rings [18], T-shaped spin filters [19] and dots connected to Luttinger liquid leads [20]. In [14, 15, 16] the QD's are attached to two leads (right and left), which we call the substitutional configuration and the transport properties are complementary to the case studied here.

In this report we combine the standard physics of the Coulomb blockade with the additional interference of the Fano type 21]. The latter is essential only in the limit of a single conducting channel, which is weakly coupled to a side quantum dot [22, 23]. The transport properties of such setup has been studied in the work by Kang et al. 24]. It was shown that the dependence of the channel conductance on the gate voltage applied to the dot is opposite to the conductance of the dot itself. Indeed the single-particle resonance locks the channel giving rise to a resonant dip of zero conductance. Therefore, at the Coulomb blockade regime the channel conductance should reveal a sequence of sharp dips as the function of the gate voltage. Similarly at very low temperature the Kondo effect should lead to the appearance of a shallow and broad valley in the conductance versus the gate voltage curve, which corresponds to the odd number of electrons on the dot.

If the spin degeneracy is lifted, say, by an applied magnetic field, and the splitting exceeds the temperature, the resonances for spin up and spin down electrons become well separated in the absence of the Kondo effect. This gives rise to the resonance spin polarized conductance due to the effect of Fano interference.

\section{THE MODEL}

In what follows we are dealing essentially with the single-level Anderson impurity model 25]. We take advantage of recently studied side dot model 23, 24] characterized by the one-dimensional Hamiltonian:

$$
\begin{aligned}
H & =-t \sum_{n, \sigma}\left(c_{n, \sigma}^{\dagger} c_{n-1, \sigma}+c_{n, \sigma}^{\dagger} c_{n+1, \sigma}\right)+\sum_{\sigma} \varepsilon_{0, \sigma} n_{\sigma} \\
& +\sum_{\sigma}\left(V d_{\sigma}^{\dagger} c_{0, \sigma}+V^{\star} c_{0, \sigma}^{\dagger} d_{\sigma}\right)+U n_{\uparrow} n_{\downarrow},
\end{aligned}
$$

where $n_{\sigma}=d_{\sigma}^{\dagger} d_{\sigma}$. The $c$-operators describe the propagation of electrons in the conducting channel, while the $d$ operators describe the occupation of the side dot attached to site zero of the channel. The dot is modeled as an Anderson impurity with a Hubbard interaction constant $U>0$ [25]. In this paper we consider $t=V=1$ 
and $\varepsilon_{F}=0$ (half-filling) for all numerical computations. We also assume that the local magnetic field induces a Zeemann splitting of the dot levels

$$
\varepsilon_{0, \uparrow}=\varepsilon_{0}+\Delta / 2, \quad \varepsilon_{0, \downarrow}=\varepsilon_{0}-\Delta / 2,
$$

and the position of the resonant level $\varepsilon_{0}$ can be adjusted by an external gate voltage.

The considered model falls into the class of Anderson Hamiltonians 26]. Let us emphasize that the conductance depends crucially on the geometry of the coupling between the dot and the channel unlike the thermodynamic properties of the model 23], such as the Kondo temperature $T_{K}$, etc. Below we are mostly concerned with the ground state properties at zero temperature.

\section{FANO RESONANCE FOR $U=0$}

Let us consider first the case $U=0$. Then the dimensionless conductance per spin channel $g_{\sigma}$ is linked to the transmission coefficient $\tau$ by the Landauer formula

$$
g_{\sigma}=\left|\tau_{\sigma}\right|^{2} .
$$

For $U=0$ the spin up and spin down electrons do not interact and we can suppress the index $\sigma$. The transmission can be computed within the one-particle picture for an electron moving at the Fermi energy $\varepsilon_{F}$ :

$$
\begin{aligned}
-\varepsilon_{F} \phi_{i} & =t\left(\phi_{n-1}+\phi_{n+1}\right)+V^{\star} \varphi \delta_{n 0}, \\
-\varepsilon_{F} \varphi & =-\varepsilon_{0, \sigma} \varphi+V \phi_{0},
\end{aligned}
$$

where $\delta_{n m}$ is the Kronecker symbol, $\phi_{n}$ refers to the amplitude of a single particle at site $n$ in the conducting channel and $\varphi$ is the amplitude at the side dot.

The scattering problem is solved by the ansatz

$$
\phi_{n<0}=e^{i q n}+\rho e^{-i q n}, \quad \phi_{n>0}=\tau e^{i q n},
$$

with the usual dispersion relation $\varepsilon=-2 t \cos q$. Substituting Eq. (5) in Eq. (4) we readily find

$$
\tau=\frac{2 i t \sin q}{2 i t \sin q+|V|^{2}\left(\varepsilon_{0}-\varepsilon_{F}\right)^{-1}},
$$

hence the conductance

$$
g_{\sigma}=\left|\tau_{\sigma}\right|^{2}=\left[1+\frac{\Gamma^{2}}{4\left(\varepsilon_{0, \sigma}-\varepsilon_{F}\right)^{2}}\right]^{-1},
$$

where $\Gamma=2|V|^{2} /\left|v_{F}\right|$ with $v_{F}=d \varepsilon / d q \mid \varepsilon_{F}$ is the Fermi velocity.

On the other hand the mean occupation number on the dot at zero temperature $\left\langle n_{\sigma}\right\rangle$ is related to the scattering phase shift by the Friedel sum rule [25, 27]

$$
\left\langle n_{\sigma}\right\rangle=-\frac{1}{\pi} \operatorname{Im} \ln \left(\varepsilon_{\mathrm{F}}-\varepsilon_{0, \sigma}+\mathrm{i} \Gamma / 2\right)
$$

suggesting the relation

$$
g_{\sigma}=\cos ^{2} \pi\left\langle n_{\sigma}\right\rangle .
$$

This relation has a geometric origin and actually holds for arbitrary $U$ provided zero temperature limit. This is in contrast to Eqs. (6)7] which are applicable only for $U=0$.

The symmetric form of the Fano resonance given by Eq. (7) is based on the symmetry of the coupling term in the Hamiltonian (1). For nonzero magnetic field $\Delta \gg \Gamma$ the two Fano resonances for spin up and spin down electrons are energetically separated. Therefore, the current through the channel is completely polarized at $\varepsilon_{F}=\varepsilon_{0, \uparrow}$ and $\varepsilon_{F}=\varepsilon_{0, \downarrow}$.

\section{FANO RESONANCE FOR $U \neq 0$ AT THE DEGENERATE LEVEL $\Delta=0$}

Let us consider the case of zero magnetic field $\Delta=0$ but finite Coulomb interaction $U$. For zero coupling, $V=$ 0 , the single particle excitations on the dot are located at energies $\varepsilon_{0}$ and $\varepsilon_{0}+U$. When the dot is weakly connected to the conducting channel (and at very low temperatures, $T<T_{K}$ ), a resonant peak in the density of states on the dot arises at the Fermi energy due to the Kondo effect. By using an approach identical to that of Refs. [9, 10], we conclude that the channel conductance $g_{\sigma}$ is controlled entirely by the expectation value of the particle number $\left\langle n_{\sigma}\right\rangle$ as given by Eq. (9). This reasoning justifies Eq. (9) for any values of $U$ and $\Delta$ 28]. Note that in the standard setup the dot's conductance is proportional to $\sin ^{2}\left(\pi\left\langle n_{\sigma}\right\rangle\right)$ [9, 10, 27] due to the different geometry of the underlying system.

If the resonant level is spin degenerate $\Delta=0$ one has $\left\langle n_{\uparrow}\right\rangle=\left\langle n_{\downarrow}\right\rangle$ independent of the value of $U$. The mean occupation of the $\operatorname{dot}\left\langle n_{\sigma}\right\rangle$ is a monotonous function of $\varepsilon_{0}$, ranging from 1 for $\varepsilon_{0} \ll \varepsilon_{F}$ to 0 for $\varepsilon_{0} \gg \varepsilon_{F}+U$. According to Eq. (9) the channel conductance vanishes for $\left\langle n_{\sigma}\right\rangle=1 / 2$.

In order to compute $\left\langle n_{\sigma}\right\rangle$ for finite $U$ we use the numerical techniques developed in Ref. 23. We compare our results to those obtained from the exact solution for $\left\langle n_{\sigma}\right\rangle$ by Wiegmann and Tsvelick 29]. The latter holds only for the linearized spectrum (which is not a serious restriction at half-filling) and has a compact form only in the case of zero magnetic field.

Numerically we obtain $\left\langle n_{\sigma}\right\rangle$ by integrating the density of states at the dot over the energy. This quantity has been previously calculated using a combined method. In the first place we consider an open finite cluster of $N=9$ sites which includes the impurity. This cluster is diagonalized using the Lanczos algorithm 30 . We then embed the cluster in an external reservoir of electrons, which fixes the Fermi energy of the system, attaching two semi-infinite leads to its right and left 32]. This is 

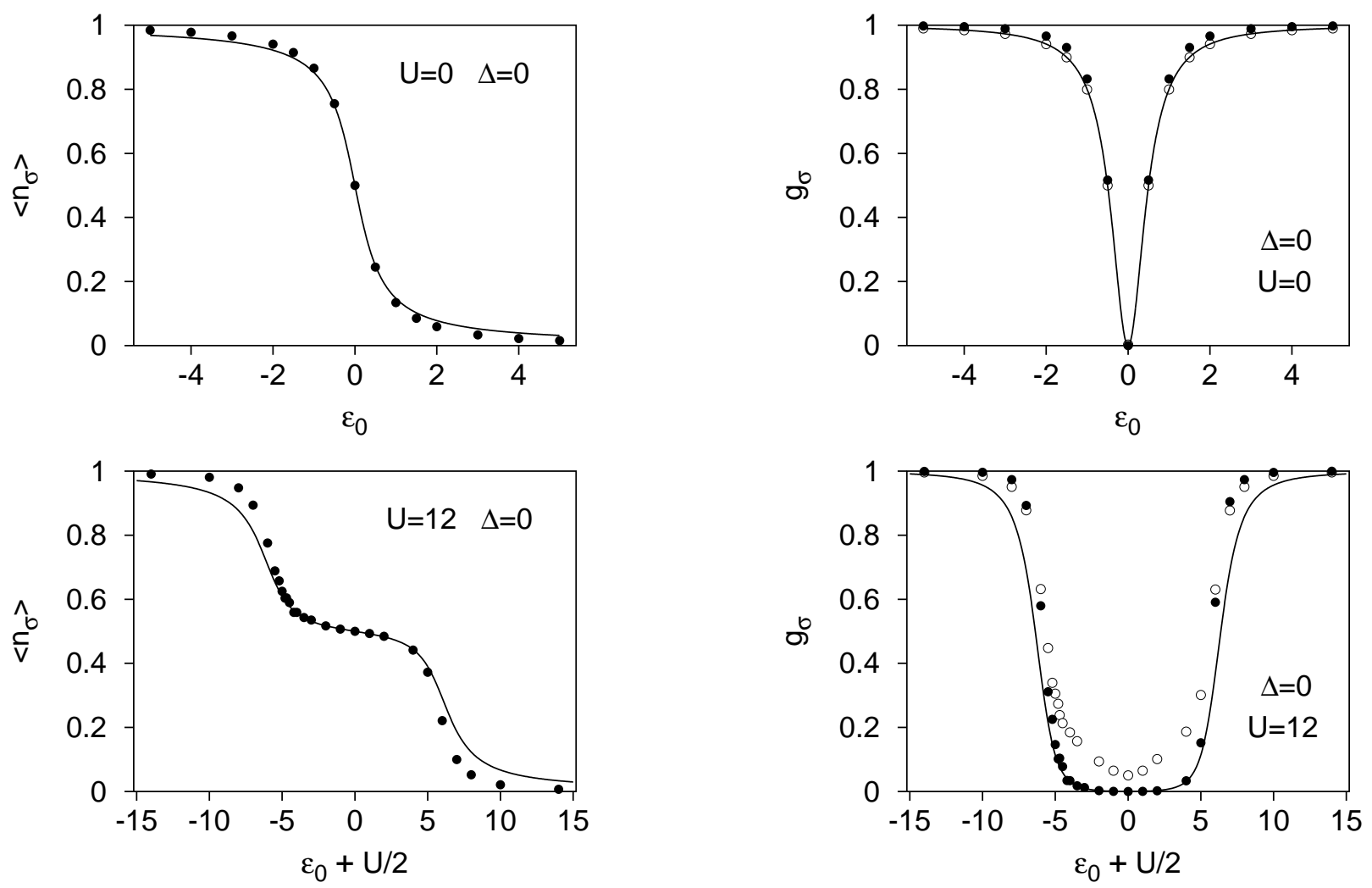

FIG. 1: The dependence of $\left\langle n_{\sigma}\right\rangle$ on the position of the resonance level $\varepsilon_{0}$ for zero magnetic field, $U=0$ (top) and $U=12$ (bottom). The results of the numerical calculation (black dots) are compared to the exact results by Wiegmann and Tsvelick [29] (solid line).

done by calculating the one-particle Green's function $\hat{G}$ of the whole system within the chain approximation of a cumulant expansion 31] for the dressed propagators. This leads to the Dyson equation $\hat{G}=\hat{G} \hat{g}+\hat{T} \hat{G}$, where $\hat{g}$ is the cluster Green's function obtained by the Lanczos method. Following Ref. [32], the charge fluctuations inside the cluster are taken into account by writing $\hat{g}$ as a combination of $n$ and $n+1$ particles with weights $1-p$ and $p$ respectively: $\hat{g}=(1-p) \hat{g}_{n}+p \hat{g}_{n+1}$. The total charge of the cluster and the weight $p$ are calculated by solving the following equations

$$
\begin{aligned}
Q_{c} & =n(1-p)+(n+1) p \\
Q_{c} & =-\frac{1}{\pi} \int_{-\infty}^{\varepsilon_{F}} \sum_{i} \operatorname{Im} G_{i i}(\varepsilon) d \varepsilon
\end{aligned}
$$

where $i$ counts the cluster sites. Once convergence is reached, the density of states is obtained from $\hat{G}$. One should stress, however, that the method is reliable only if the cluster size is larger then the Kondo length.

We also perform the alternative calculation of the conductance evaluating the local density of states $\rho_{\sigma}(\varepsilon)$ at

FIG. 2: The channel conductance $g_{\sigma}$ versus the resonance energy $\varepsilon_{0}$ for zero magnetic field, $U=0$ (top) and $U=12$ (bottom). The conductance computed numerically by different means from Eq. (11) (white dots) as well as from Eq. (9) (black dots) is compared to the conductance obtained from the exact solution by Wiegmann and Tsvelick [29] (solid line).

site $0 \underline{33}$

$$
g_{\sigma}=2 \pi t \rho_{\sigma}\left(\varepsilon_{F}\right)
$$

We plot $\left\langle n_{\sigma}\right\rangle$ versus the resonance level energy $\varepsilon_{0}$ for $U=0$ and $U=12$. In the presence of Coulomb repulsion the mean occupancy of the $\operatorname{dot}\left\langle n_{\sigma}\right\rangle$ has a flat region near the value $1 / 2$ due to the Kondo effect. This corresponds to a valley of small channel conductance (see Fig. 2). Note that similar results for the limit $U \rightarrow \infty$ have been recently obtained by Bulka and Stefanski [34].

The conductance found from the numerical evaluation of $\left\langle n_{\sigma}\right\rangle$ for finite $U$ is in better agreement with the exact results 29] than the conductance computed from Eq. (11). Presumably, the discrepancy originates from the finite-size effects (the Kondo length is larger than the system size used in the numerical computation). The numerical calculation of $\left\langle n_{\sigma}\right\rangle$, which involves the energy integration, appears to be more precise then that of the local density $\rho_{\sigma}\left(\varepsilon_{F}\right)$. 

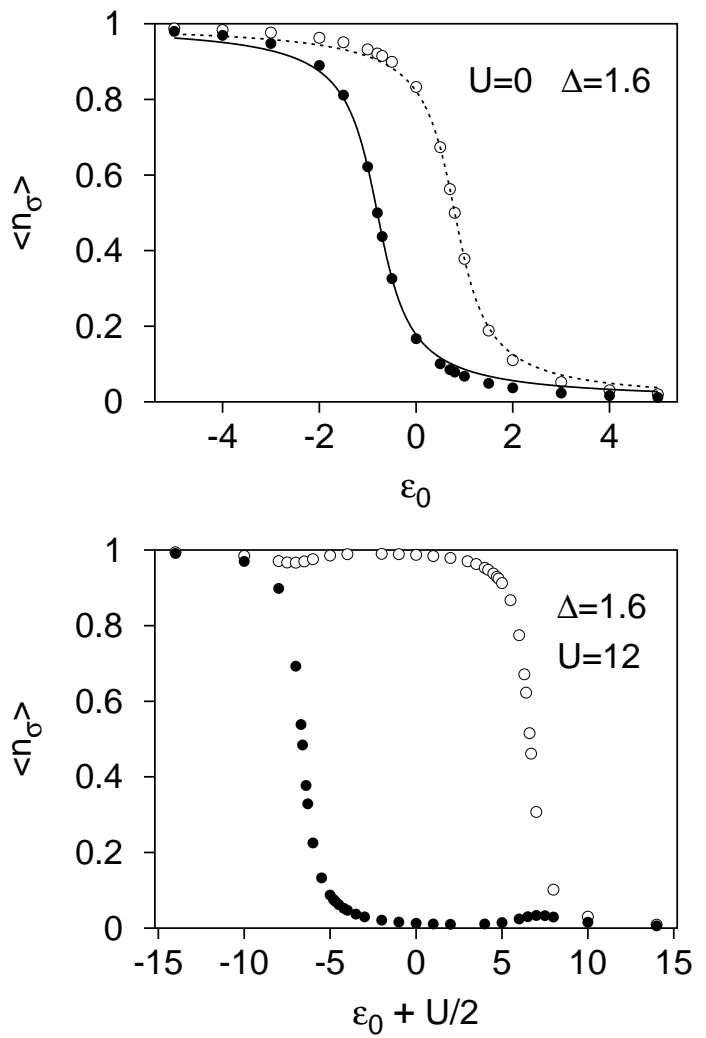

FIG. 3: The dependence of $\left\langle n_{\sigma}\right\rangle$ on the position of the resonance level $\varepsilon_{0}$ for a finite splitting $\Delta=1.6, U=0$ (top) and $U=12$ (bottom). The black (white) dots represent the numerical results for the spin up (spin down) occupation number. The solid and the dashed line on the top figure represent the exact results of Eq. (7).

\section{SPIN FILTER FOR $U \neq 0$ AND $\Delta \neq 0$}

In the presence of a magnetic field the dot occupation for spin up and spin down electrons become different. At zero temperature the conductance in each spin channel can be found from Eq. (9). The expectation value $\left\langle n_{\sigma}\right\rangle$ decreases from 1 to 0 with increasing the resonant energy $\varepsilon_{0}$, but it takes the value $1 / 2$ at different gate voltages for $\sigma=\uparrow$ and $\sigma=\downarrow$ (see Fig. [3).

Consequently we may obtain a perfect spin filter as in the case of noninteracting electrons. In Fig. 4 we plot the numerical results for the conductance obtained from Eqs. (9) and (11) in a similar manner as in Fig. 2 for $U=0$ and $U=12$ with $\Delta=1.6$.

Since the dot levels are no longer degenerate the Kondo effect is totally suppressed even at zero temperature. Indeed no plateaus formed near $\left\langle n_{\sigma}\right\rangle=1 / 2$ in Fig. 3. As the result the spin-dependent resonances in the conductance have the width $\Gamma$. The effect of large $U$ manifests itself mainly in the mere enhancement of the level splitting. At low temperatures $T \ll \Delta$ the mean occupation number $\left\langle n_{\downarrow}\right\rangle$ is close to unity while the occupation num-
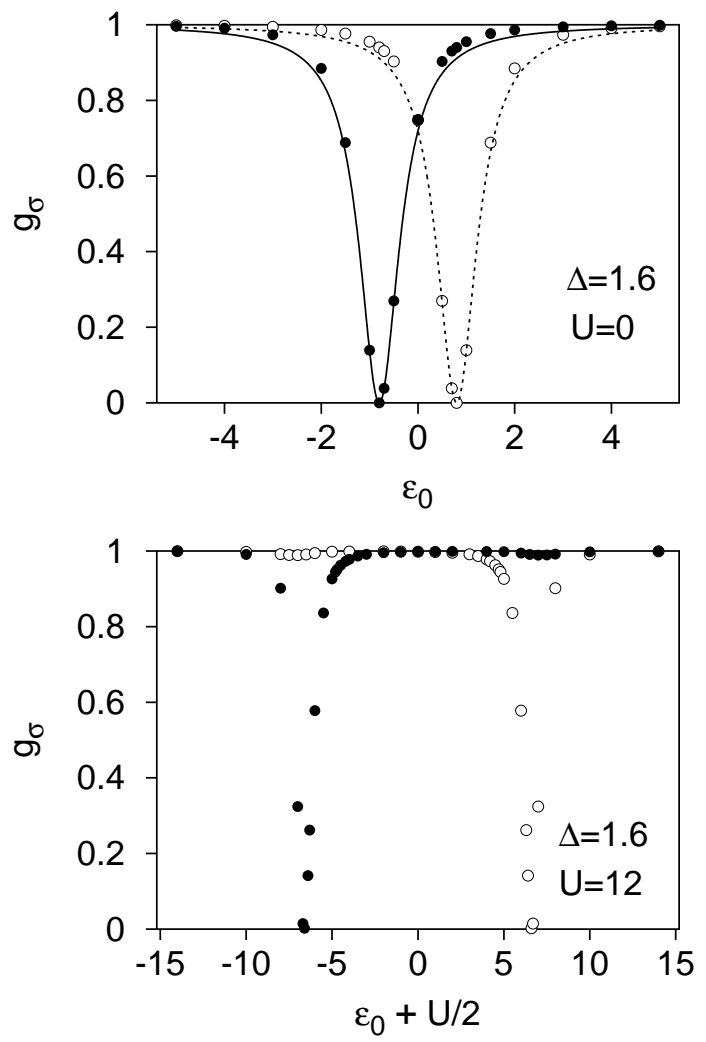

FIG. 4: The channel conductance $g_{\sigma}$ versus the resonance energy $\varepsilon_{0}$ for finite splitting $\Delta=1.6, U=0$ (top) and $U=12$ (bottom). The conductance is computed numerically for spin up (black dots) and spin down (white dots) electrons. The solid and the dashed line on the top figure represent the exact results of Eq. (7).

ber for the opposite spin direction decreases to zero. One can observe, however, from Fig. 3 that there is a mixing of the levels which gives rise to the non-monotonous behavior of $\left\langle n_{\sigma}\right\rangle$. This results in a small dip of the spin down conductance at the resonance energy for the spin up electrons and vice versa.

\section{CONCLUSIONS}

We have studied numerically the spin-dependent conductance of electrons through a single ballistic channel weakly coupled to a quantum dot. The Fano interference on the dot reverses the dependence of the channel conductance on the gate voltage compared to that of the dot's conductance. If the spin degeneracy of the dot levels is lifted, the conductance of the channel reveals Coulomb blockade dips, or anti-resonances, as a function of the gate voltage. If the level is spin degenerate the Kondo effect suppresses the channel conductance in a broad range of the gate voltages.

When the coupling between the channel and the dot is 
not truly local the resonances should become highly antisymmetric. In the idealized picture of the single ballistic channel transport the zero temperature conductance vanishes at the resonance values of the gate voltage due to the Fano interference. If there is no spin degeneracy, the resonances have single-particle origin (for the simple model (1) they are separated roughly by the Coulomb energy $U+\Delta)$. At the resonance the channel conductance for a certain spin direction changes abruptly from its ballistic value $e^{2} / h$ to zero, while the opposite spin conductance remains approximately at the ballistic value.

This is in contrast with previous calculations that considered a substitutional QD, where the complementary effect is found, i.e., a perfect conductance for one spin species. However, in these cases due to the local correlations and hibridization with the leads, there remains a finite conductance of the opposite spin species blurring the filtering condition. In the lateral dot case considered here we find total suppression of one spin species, thus leading to a perfect spin filter. Moreover we obtain that the energetical splitting of the Fano resonances for spin up and spin down electrons is of the order of the Coulomb interaction $U$ for large $U$ rather than of the order of the Zeeman splitting $\Delta$ as it would be for noninteracting electrons $U=0$. Thus a strong electron interaction on the dot (realistic values for $U$ are of the order of $1 \mathrm{meV}$ ) will allow to apply very weak magnetic fields (less than 1Tesla) and still observe a significant splitting of the Fano resonances (provided $k_{B} T<\Delta$ ), and thus a high quality spin filter. This is because not only the spin filter is totally reflecting electrons with the desired spin $\sigma$, but it will be then also close to fully transparent for electrons with opposite spin. Note, however, that the coupling to the dot should preserve the phase coherence and there should be only one conducting channel.

We thank A. Aharony, O. Entin-Wohlman, V. Fleurov, L. Glazman, K. Kikoin and M. Raikh for helpful discussions. K.H and M.E.T acknowledge CONICET for support. Part of this work was done under projects Fundación Antorchas 14116-168 and 14116-212.

[1] Mesoscopic Electron Transport, L. L. Sohn, L. P. Kouwenhoven, and G. Schön, eds, (Kluwer, Dordreht, 1997).

[2] Mesoscopic Quantum Physics, E. Akkermans, G. Montambaux, J. L. Pichard, and J. Zinn-Justin, eds, (North Holland, Amsterdam, 1995).

[3] Mesoscopic phenomena in Solids, B. L. Altshuler, P. A. Lee, and R. A. Web, eds, (North Holland, Amsterdam, 1991).

[4] I. L. Aleiner, P. W. Brouwer, and L. I. Glazman, Physics Reports, 358, 309 (2002).
[5] Y. Alhassid, Rev. Mod. Phys., 72, 895 (2000).

[6] L. P. Kouwenhoven, C. M. Marcus, P. L. McEuen, S. Tarusha, R. M. Westerweld, and N. S. Wingreen, in Ref. [1].

[7] D. V. Averin and K. K. Likharev in Ref. [3].

[8] Leo Kouwenhoven and Leonid Glazman, Physics World, 14, 33 (2001).

[9] L. I. Glazman and M. E. Raikh, Sov. Phys. JETP Lett., 47, 454 (1988).

[10] T. K. Ng and P. A. Lee, Phys. Rev. Lett., 61, 1768 (1988).

[11] D. Goldhaber-Gordon, H. Shtrikman, D. Mahalu, D. Abush-Madger, U. Meirav, M. A. Kastner, Nature, 391, 156 (1998).

[12] S. M. Cronenwet, T. H. Oosterkamp, am nd L. P. Kouwenhoven, Science 281, 540 (1998).

[13] J. Schmid, J. Weis, K. Eberl, and K. von Klitzing, Physica B, 256, 182 (1998).

[14] T. Costi, Phys. Rev. B, 64, 241310(R) (2001).

[15] P. Recher, E. Sukhorukov and D. Loss, Phys. Rev. Lett., 85, 1962 (2000).

[16] T. K. Ng and P. A. Lee, Phys. Rev. Lett., 61, 1768 (1988).

[17] Y. Ohno, D. K. Young, B. Beschoten, F. Matsukura, H. Ohno, D. D. Awschalom, Nature, 402, 790 (1999).

[18] M. Popp, D. Frustaglia and K. Richter, preprint, cond-mat/0301064 (2003).

[19] A. Kiselev and K. W. Kim, preprint, cond-mat/0203261 (2002).

[20] D. Schmeltzer, A. R. Bishop, A. Saxena, and D. L. Smith, Phys. Rev. Lett., 90, 116802 (2003).

[21] U. Fano, Phys. Rev., 124, 1866 (1961); J. A. Simpson and U. Fano, Phys. Rev. Lett., 11, 158 (1963).

[22] J. Göres, D. Goldhaber-Gordon, S. Heemeyer, M. A. Kastner, H. Shtrikman, D. Mahalu, and U. Meirav, Phys. Rev. B, 62, 2188 (2000).

[23] M. E. Torio, K. Hallberg, A. H. Ceccatto, and C. R. Proetto, Phys. Rev. B, 65, 085302 (2002).

[24] K. Kang, Y. Cho, Ju-Jin Kim, and Sung-Chul Shin, Phys. Rev. B, 63, 113304 (2001).

[25] A. C. Hewson, The Kondo Problem to Heavy Fermions (Cambridge University Press, Cambridge UK 1993).

[26] P. W. Anderson, Phys. Rev., 124, 41 (1961).

[27] D. C. Langreth, Phys. Rev., 150, 516 (1966).

[28] For a detailed derivation in the absence of magnetic fields we refer to Ref. [24].

[29] P. B. Wiegmann and A. M. Tsvelick, J. Phys. C, 16, 2281 (1983).

[30] E. Dagotto and A. Moreo, Phys. Rev. D, 31, 865 (1985); E. Gagliano, E. Dagotto, A. Moreo and F. Alcaraz, Phys. Rev. B, 34, 1677 (1986).

[31] C. Caroli, R. Combesco, P. Nozieres, and F. Alcaraz, J. Phys. C, 4, 916 (1971); W. Metzner, Phys. Rev. B, 43, 8549 (1991).

[32] V. Ferrari, G. Chiappe, E. V. Anda, and M. A. Davidovich, Phys. Rev. Lett., 82, 5088 (1999).

[33] Y. Meir and N. S. Wingreen, Phys. Rev. Lett., 68, 2512 (1992).

[34] B. R. Bulka and P. Stefanski, Phys. Rev. Lett., 86, 5128 (2001). 